\title{
miR-140-3p Suppresses Cell Growth And Induces Apoptosis In Colorectal Cancer By Targeting PD-LI
}

This article was published in the following Dove Press journal: OncoTargets and Therapy

\author{
Wei Jiang ${ }^{1,2, *}$ \\ Tao $\mathrm{Li}^{1,2, *}$ \\ Jingjing Wang $\mathbb{D}^{1,2}$ \\ Ruonan Jiao (iD) 1,2 \\ Xiao Shi ${ }^{1,2}$ \\ Xiaodan Huang ${ }^{1,2}$ \\ Guozhong $\mathrm{Ji}^{1,2}$ \\ 'Department of Gastroenterology, The \\ Second Affiliated Hospital of Nanjing \\ Medical University, Nanjing 2100II, \\ People's Republic of China; ${ }^{2}$ Jiangsu Key \\ Laboratory of Cancer Biomarkers, \\ Prevention And Treatment, Collaborative \\ Innovation Center For Cancer \\ Personalized Medicine, Nanjing Medical \\ University, Nanjing 211166, People's \\ Republic of China \\ *These authors contributed equally to \\ this work
}

Correspondence: Guozhong Ji

Department of Gastroenterology, The Second Affiliated Hospital of Nanjing

Medical University, Nanjing 2100I I,

People's Republic of China

Email jgz@njmu.edu.cn
Background: A variety of miRNAs have been recently reported to be abnormally expressed in colorectal cancer (CRC). A growing number of studies have demonstrated that aberrantly expressed miRNAs are closely related to the development and progression of CRC. It has been found that miR-140-3p plays a vital role in several cancers. However, its expression, roles and mechanisms in CRC are remain unknown.

Materials and methods: Reverse transcription-quantitative polymerase chain reaction (RTqPCR) were used to determine miR-140-3p expression in CRC tissues and cell lines. CCK8, migration, invasion and flow cytometric assays were used to determine the influence of miR-140$3 p$ upregulation on cell proliferation, migration, invasion and apoptosis of CRC cells. Luciferase reporter assays and Western blots were utilized to identify the target genes of miR-140-3p. In addition, the potential mechanism of miR-140-3p action in CRC cells was elucidated.

Results: In our study, miR-140-3p expression was significantly decreased in CRC tissues and cell lines. Overexpression of miR-140-3p attenuated proliferation, migration, and invasion and induced the apoptosis of CRC cells. Bioinformatics analyse and luciferase reporter analysis identified PD-L1 as a putative target gene of miR-140-3p. PD-L1 was overexpressed in CRC tissues and inversely correlated with miR-140-3p expression. Suppression of PD-L1 expression in CRC cells generated biological behaviours in CRC cells that were similar to those observed after treated with miR-140-3p mimics. Restoration of PD-L1 expression partially attenuated the inhibitory effect of miR-140-3p on CRC cells. Western blot were used to verify the effect of PD-L1 expression on PI3K/AKT pathway. In addition, overexpression of miR-140-3p could inhibit CRC tumor growth in vivo.

Conclusion: In general, these data demonstrate that miR-140-3p acts as a tumour suppressor in CRC by directly targeting PD-L1 and inactivating PI3K/AKT pathway, suggesting that miR-140-3p might be a novel target for CRC diagnosis and treatment.

Keywords: microRNA-140-3p, PD-L1, colorectal cancer, apoptosis

\section{Introduction}

Colorectal cancer (CRC) is the third most common cancer and the third most common cause of cancer related deaths in the world. ${ }^{1}$ Currently, the surgical treatment of $\mathrm{CRC}$ is effective for patients diagnosed early. However, due to its high recurrence and metastasis rate, approximately $70 \%$ of patients have metastases at the time of diagnosis. ${ }^{2}$ Colorectal cancer diagnosis and therapy interventions have achieved some progress in the past decade. However, the 5-year survival rate for advanced colorectal cancer is approximately $10 \%{ }^{3}$ Hence, more suitable therapeutic targets and their underlying mechanisms should be explored. 
microRNAs (miRNAs) are a group of single stranded RNAs of approximately 18-24 nucleotides in length that are highly conversed and non-coding. miRNAs can regulate gene expression through binding to the $3^{\prime}$ untranslated regions (3'UTRs) of RNA transcripts, resulting in mRNA degradation or translation inhibition. Several studies have revealed that miRNAs could be pivotal regulators of tumor suppressors or oncogenes. ${ }^{4,5}$ In addition, miRNAs could also play vital role in various biological processes such as cell proliferation, apoptosis and the cell cycle. ${ }^{6}$ A growing number of studies have demonstrated that aberrantly expressed miRNAs are tightly linked to CRC occurrence and progression, so these miRNAs may be considered as novel promising targets for CRC diagnosis. ${ }^{7}$ MicroRNA-140-3p (miR-140-3p) has been abnormally expressed in many different cancers such as lung carcinoma, breast cancer, spinal chordoma and hepatocellular carcinoma. ${ }^{8-11}$ However, the expression, biological function and underlying mechanisms of miR-140-3p in CRC remain unclear.

Many researchers have shown the overexpression of programmed cell-death 1 ligand 1 (PD-L1) in various tumours including CRC. ${ }^{12-14}$ Furthermore, studies have shown that PD-L1 expression levels are negatively correlated with the prognosis of patients and the malignancy degree of tumours. ${ }^{15}$ As an immune escape mechanism, PD-L1 expressed on the surface of tumour cells can bind to PD-1 on T cells, which can inhibit the activation, proliferation and survival of $\mathrm{T}$ cells and enhance the immune evasion of tumour cells. ${ }^{16}$ In this study, we found that miR-140-3p was noticeably downregulated in CRC cell lines and CRC tissues. PD-L1 expression was negatively correlated with miR-140-3p expression in CRC tissues. miR-140-3p may act as a tumour suppressor through directly targeting PD-L1. Overexpression of miR-140-3p markedly inhibited cell proliferation, invasion, and migration and induced apoptosis of CRC cells by repressing PDL1. miR-140-3p inhibits in vitro and in vivo activation of the PI3K/AKT pathway. Hence, miR-140-3p might be a novel target for CRC diagnosis and treatment.

\section{Materials And Methods}

\section{Patients And Tissues}

In this study, thirty-one paired colorectal cancer (CRC) tissues and adjacent non-tumour tissues were collected by surgical resection at the second afflicted hospital of Nanjing Medical University. No patients received radio or chemotherapy before surgery. All samples were frozen directly in liquid nitrogen and deposited at $-80{ }^{\circ} \mathrm{C}$. The research was approved by the Ethics Committee of the second afflicted hospital of Nanjing Medical University. Informed consent was signed by all the patients.

\section{Cell Lines}

Two human CRC cell lines, HCT116 and SW480, and the normal human colonic epithelial cell line NCM460 were purchased from the Institute of Biochemistry and Cell Biology (SIBCB) of the Chinese Academy of Sciences (Shanghai, China). All cell lines were cultured in DMEM containing 10\% FBS, 100 units $/ \mathrm{mL}$ penicillin and 100 units $/ \mathrm{mL}$ streptomycin (Thermo Fisher Scientific, Waltham, MA, USA) in $5 \% \mathrm{CO}_{2}$ at $37^{\circ} \mathrm{C}$.

\section{Plasmid Transfection}

miR-140-3p miRNA mimics(miR-140-3p), a miRNA mimics negative control (miR-NC),a PD-L1 siRNA and a siRNA negative control (si-NC)were synthesized by Shanghai GenePharma Co., Ltd. (Shanghai, China). The PD-L1 overexpression plasmid PCMV-PD-L1 and the empty plasmid PCMV were constructed by Hai GenePharma Co., Ltd. (Shanghai, China).The PD-L1 siRNA sequences were 5'GCCGAAGUCAUCUGGACAATT-3', and the NC siRNA sequences were: 5'-UUCUCCGAACGUGUCACGUTT-3'. Lipofectamine 2000 (Invitrogen, USA) was used to transfect cells according to the manufacturer's instructions.

\section{RNA Isolation And RT-qPCR}

Total RNA was isolated from tissues or cells using TRIzol (Invitorgen, USA) according to the manufacturer's protocol. First, cDNA was generated using a miRNA First Strand cDNA Synthesis Kit (Vazyme, China), and then, the expression of miR-140-3p was evaluated by using a miRNA Universal SYBR Master Mix (Vazyme, China), which was analysed on an ABI 7500 QPCR (Thermo Fisher Scientific, USA). In addition, total RNA was also reverse transcribed to generate cDNA utilizing the HiScript 1st Strand cDNA Synthesis Kit (Vazyme, China) and qPCR was performed using HiScript QRT SuperMix for qPCR (Vazyme, China) to detect PD-L1 expression. The primers were as follows:

Forward primer for miR-140-3P: 5'GCGCGTACCACA GGGTAGAA3';

Reverse primer for miR-140-3P: 5'AGTGCAGGGTC CGAGGTATT3';

Forward primer for PD-L1:5'TGGCATTTGCTGAAC GCATTT3'; 
Reverse primer for PD-L1:5'TGCAGCCAGGTCTAA TTGTTTT3'.

Relative gene expression was determined using the $2^{-\Delta \Delta \mathrm{Ct}}$ method.

\section{Cell Proliferation Assay}

Cell proliferative ability was assessed using a Cell Counting Kit8 (DojinDo $\square$ Japan). A total of $2 \times 10^{3}$ transfected cells/ well were resuspended and plated in 96-well culture plates. Cell viability was determined every day following the manufacturer's protocol. For the adherent colony formation assay, $1 \times 10^{3}$ transfected cells/well were seeded in $35 \mathrm{~mm}$ culture plates at $37{ }^{\circ} \mathrm{C}$ in $5 \% \mathrm{CO}_{2}$. After 14 days, colonies were fixed with $4 \%$ paraformaldehyde and stained with $0.1 \%$ crystal violet. Colony formation was determined by counting the number of stained colonies.

\section{Cell Migration And Invasion Assay}

For the migration assay, $2.5 \times 10^{4}$ cells in serum-free medium were seeded on the upper side of $8 \mu \mathrm{m}$-pore-size transwell chamber (Millipore, USA) 48 hrs after transfection. Medium containing 20\% FBS was added to the lower chamber. After $48 \mathrm{hrs}$, the cells on the upper side of the membranes were removed carefully using a cotton swab while the invasive cells were fixed with methanol, stained with $0.1 \%$ crystal violet and counted under an inverted microscope (Olympus, Japan).The invasion assay procedure was similar to the migration assay, except that the porous polycarbonate membranes of transwell chambers were spread with Matrigel (Corning, USA) prior to the addition of cells.

\section{Flow Cytometric Analysis}

HCT116 and SW480 cells were transfected for $48 \mathrm{hrs,}$ harvested by trypsinization and then washed twice with cold PBS. Cells were subsequently double stained with FITC-labelled Annexin V and propidium iodide by using the FITC Annexin V Apoptosis Detection Kit (BD Biosciences, USA) according to the manufacturer's instructions. The percentage of apoptotic cells was determined by flow cytometry. (FACS Calibur; BD Biosciences, USA).

\section{Western Blot Analysis}

Total protein was extracted from tissue or cultured cells using RIPA (Beyotime, China) containing a protease inhibitor cocktail (Roche, USA). Protein concentrations were assessed using a BCA Protein Assay Kit (Beyotime, China). Equal amounts of protein were separated by $10 \%$ SDS-PAGE and transferred to polyvinylidene fluoride membranes (Millipore, USA). After blocking the membranes with 5\% nonfat milk, different specific antibodies were incubated with the membrane. The antibodies used were as follows: anti-PD-L1 (Cell Signaling Technology, 3127S), anti-PI3K (Santa Cruz Biotechnologies cat.no. sc-136298), anti-p-PI3K (Cell Signaling Technology, cat. no. 4228), anti-AKT (Cell Signaling Technology, 4691S), anti-p-AKT (Cell Signaling Technology, 4060S),anti-Bcl-2(Cell Signaling Technology, 15071T), anti-Bax(Cell Signaling Technology, 5023T), and anti-GAPDH (Proteintech, 60004-1-Ig). Goat anti-Mouse HRP (FDbio science, FDM007), and Goat anti-Rabbit HRP (FDbio science, FDR007). Finally, an ECL chromogenic substrate was applied for detecting the fluorescent signals (BIO-RAD, USA).

\section{Luciferase Reporter Assay}

The wild-type (WT) and mutant PD-L1-3'UTR (MUT) plasmids were constructed by GenScript (Nanjing, China). Cells were seeded in 24-well plates and grown for $24 \mathrm{hrs}$ before transfection. Lipofectamine 2000 (Invitrogen, USA) was used to cotransfect miR-140-3p mimics or the miR-NC and wild-type or mutant PD-L1-3'UTR plasmids into cells. Transfected cells were harvested after $48 \mathrm{hrs}$ and then analysed using a Dual-Luciferase Reporter Assay System (Promega, USA).

\section{In Vivo Tumour Growth Assay}

This study was carried out in accordance with the recommendations in the Guide for the Care and Use of Laboratory Animals of the National Institutes of Health. The protocol was approved by the Committee on the Ethics of Animal Experiments of the Nanjing Medical University. Six to eight-week-old BALB/c-nude mice were purchased from the Animal Experimental of the Nanjing Medical University. The mice were randomly divided into two groups ( $\mathrm{n}=5$ per group). HCT116 cells $\left(1 \times 10^{7}\right)$ that had been transfected with miR-140-3p mimics or the miR-NC were subcutaneously injected into the right flank of nude mice. Tumour volume and weight were measured every 2 days, and tumour volume was determined using the following formula: volume $=0.5 \times$ tumour width $^{2} \times$ tumour length. Twenty-eight days later, the mice were sacrificed, the neoplasms were weighed, and the proteins were harvested from tissues for Western blot analyses. 


\section{Statistical Analysis}

All data are presented as the means \pm SD. Statistical analysis was performed using SPSS 20.0. The $t$-test and one-way analysis of variance were used, all statistical tests were two-sided, and $\mathrm{p}<0.05$ was considered statistically significant.

\section{Results}

\section{miR-I40-3p Expression Is Downregulated In CRC Tissues And Cells}

To determine the expression of miR-140-3p in CRC, the expression of miR-140-3p in 31 paired human CRC tissues and adjacent normal tissues was determined by using qRTPCR in this study. As depicted in Figure 1A, the expression of miR-140-3p was significantly downregulated in CRC tissues. We next assessed miR-140-3p expression in two CRC cell lines (HCT116 and SW480) and a normal human colon epithelium cell line (NCM460), there were lower levels of miR-140$3 p$ expression in CRC cell lines than in NCM460 cells (Figure 1B). Obviously, these results indicate that decreased of miR-140-3p expression is highly correlated with CRC progression.

\section{miR-I40-3p Overexpression Inhibits Proliferation, Migration And Invasion In CRC Cells}

To evaluate the biological role of miR-140-3p in CRC, miR-140-3p mimics or miR-NC were transfected into HCT116 and SW480 cells. CCK8 assay, colony formation, migration and invasion assays were used to detect cell proliferation and migration abilities. RT-qPCR results confirmed that transfection with miR-140-3p mimics led to the

A

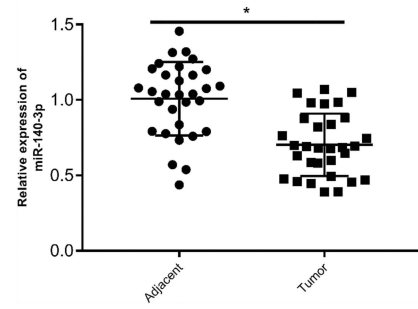

B

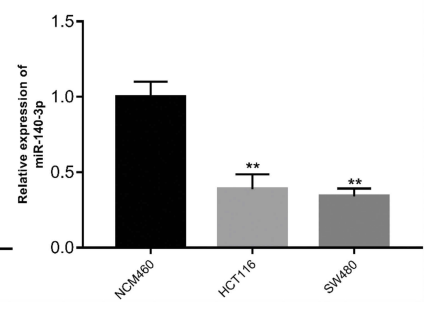

Figure I Expression of miR-140-3p is decreased in colorectal tissues and cell lines. Notes: (A) RT-qPCR analysis was performed to determine miR-140-3p expression in $3 \mathrm{I}$ pairs of colorectal cancer and adjacent non-tumour tissues. (B) Expression of miR-140-3p in two CRC cell lines (HCTII6 and SW480) and a normal human colon epithelium cell line (NCM460) was measured by RT-qPCR assay. ${ }^{*} \mathrm{P}<0.05$. Abbreviations: CRC, colorectal cancer; RT-qPCR, reverse transcription-quantitative PCR. overexpression of miR-140-3p in HCT116 and SW480 cells compared to the control (Figure 2A). As shown in Figure $2 \mathrm{~B}$, the colony formation results showed that transfection with miR-140-3p mimics decreased the colony numbers. Similarly, the CCK8 assay showed that the growth of CRC cells transfected with the miR-140-3p mimics was significantly impaired compared to the growth of cells transfected with the miR-NC (Figure 2C). In addition, migration and invasion assays indicated that the migration of CRC cells transfected with miR-140-3p mimics was notably impeded (Figure 2D and E).These results indicate that miR-140-3p overexpression could inhibit the proliferation, migration and invasion of CRC cells.

\section{Upregulation Of miR-140-3p Promotes The Apoptosis Of CRC Cells}

Flow cytometry was performed to assess the effect of miR140-3p upregulation on CRC cell apoptosis. As shown in Figure 2F, HCT116 and SW480 CRC cells exhibited similar results, and the percentage of apoptotic cells was dramatically larger in the group treated with miR-140-3p mimics than it was in the miR-NC group. In addition, we investigated Bcl-2 and Bax expression after transfecting cells with miR-140-3p. As shown in Supplementary Figure 1A, upregulation of miR-140-3p suppressed Bcl-2 expression, and increased Bax expression.These data indicated that miR140-3p promotes the apoptosis of CRC cells.

\section{PD-LI Is A Direct Target Of miR-I40-3p}

To elucidate the biological mechanisms underlying the role of miR-140-3p in CRC, we explored the potential targets of miR140-3p using TargetScan and miRWALK databases. As shown in Figure 3A, the results revealed that PD-L1 was a predicted target gene. The 3 '-UTR of PD-L1 contains a complementary site for miR-140-3p (Figure 3A). To determine whether miR-140-3p directly targets the PD-L1 3'-UTR, a luciferase reporter assay was performed. As shown in Figure 3B, overexpression of miR-140-3p decreased the luciferase activity from the wild type PD-L1 3'-UTR reporter in CRC cells. Furthermore, miR-140-3p mimics did not influence the luciferase activity of the reporter carrying the mutated PD-L1 3'-UTR (Figure 3B). To further determine whether miR-140-3p can regulate the PD-L1 expression, Western blots were used to determine the expression of PD-L1 in CRC cells after transfection with miR-140-3p mimics or the NC. The results showed that miR-140-3p mimics obviously reduced the PD-L1 expression (Figure 3C). We also 

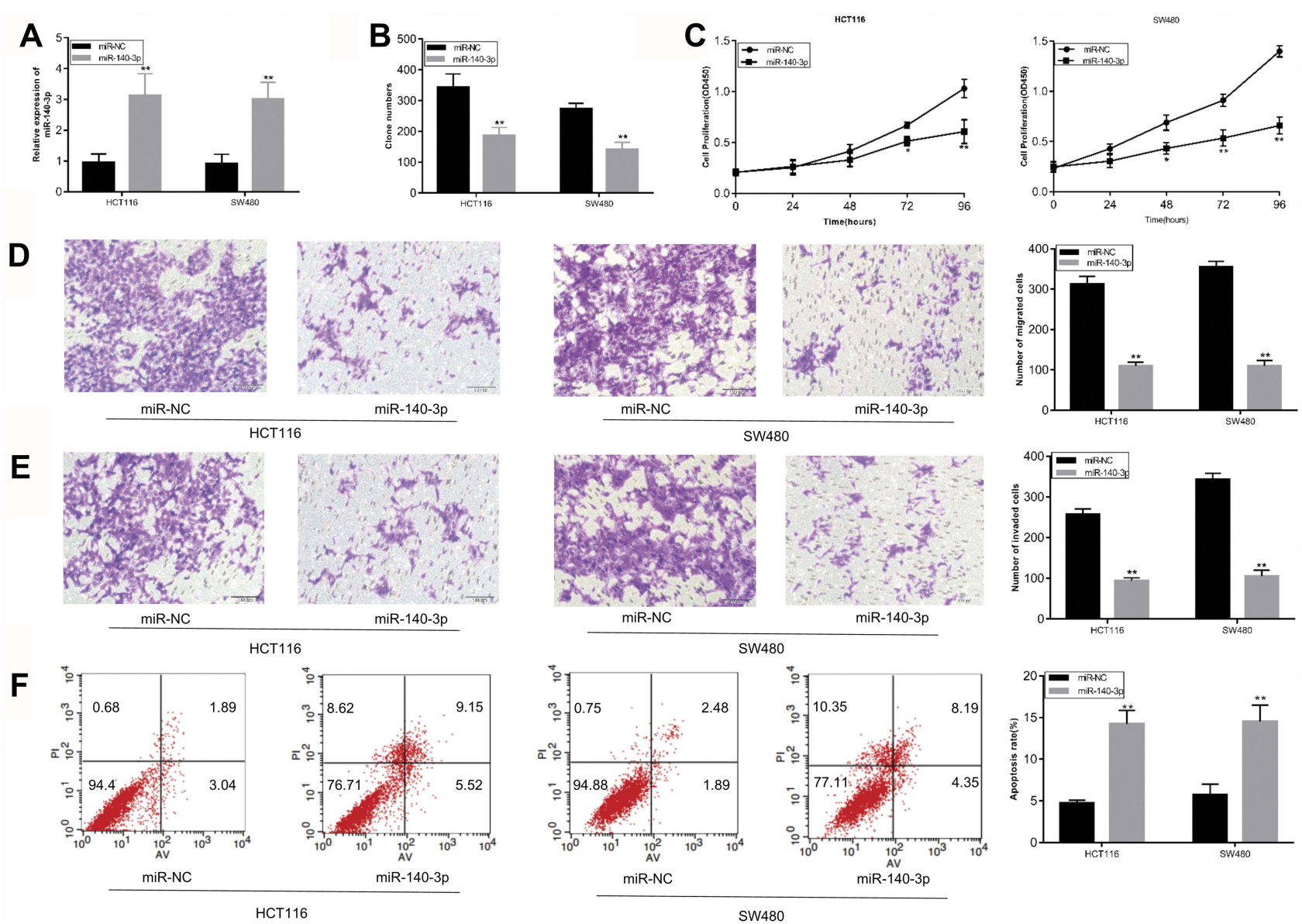

Figure 2 miR-140-3p regulates the proliferation, migration, invasion and apoptosis of HCTII6 and SW480 cells.

Notes: HCTII6 and SW480 cells were transfected with miR-I40-3p mimics or the miR-NC, and then the transfected cells were used for the following analysis. (A) The expression of miR-140-3p was detected by RT-qPCR. (B and C) After transfection with miR-I40-3p mimics or the miR-NC, colony formation and CCK8 assays were used to assess the proliferation of HCTII6 and SW480 cells. (D and E) Migration and invasion assays were used to analyse the migration and invasion abilities of HCTII 6 and SW480 cells transfected with miR-I40-3p mimics or the miR-NC. (F) Apoptosis in HCTII6 and SW480 cells transfected with miR-I40-3p mimics or the miR-NC was measured by flow cytometric analysis. $* \mathrm{P}<0.05$, ** $\mathrm{P}<0.01$.

Abbreviations: CCK8, Cell counting kit8; miR-NC, negative control miRNA mimics.

investigated PD-L1 mRNA expression in 31 paired CRC tissues and adjacent normal tissues by RT-qPCR. The results indicated that PD-L1 expression at the mRNA level was much higher in CRC tissues than it was in normal tissues (Figure 3D). Moreover, miR-140-3p levels were conversely related to PD-L1 levels in CRC tissues (Figure 3E). Taken together, these data indicate that PD-L1 is a direct target of miR-140-3p in CRC.

\section{Repression Of PD-LI Simulates The Effect Of miR-140-3p In CRC Cells}

To determine whether the role of miR-140-3p in CRC cells was mediated by PD-L1 inhibition. PD-L1 expression in HCT116 and SW480 CRC cells was knocked down by transfecting with PD-L1 siRNA. Western blot results showed that PD-L1 expression was suppressed in CRC cells after PDL1 siRNA transfection (Figure 4A). Subsequently, downregulation of PD-L1 inhibited cell proliferation (Figure 4B and C), migration (Figure 4D), and invasion (Figure 4E) of HCT116 and SW480 cells. In addition, inhibition of PD-L1 expression induced the apoptosis of CRC cells (Figure 4F). Furthermore, Western blot results showed inhibition of PD-L1 expression also suppressed Bcl-2 expression, and increased Bax expression (Supplementary Figure 1). These data not only suggest that the biological effects of PD-L1 suppression were similar to those caused by the upregulation of miR-140-3p in CRC cells, but also indicate that miR-140-3p exerted an inhibitory effect on CRC development by targeting PD-L1. 

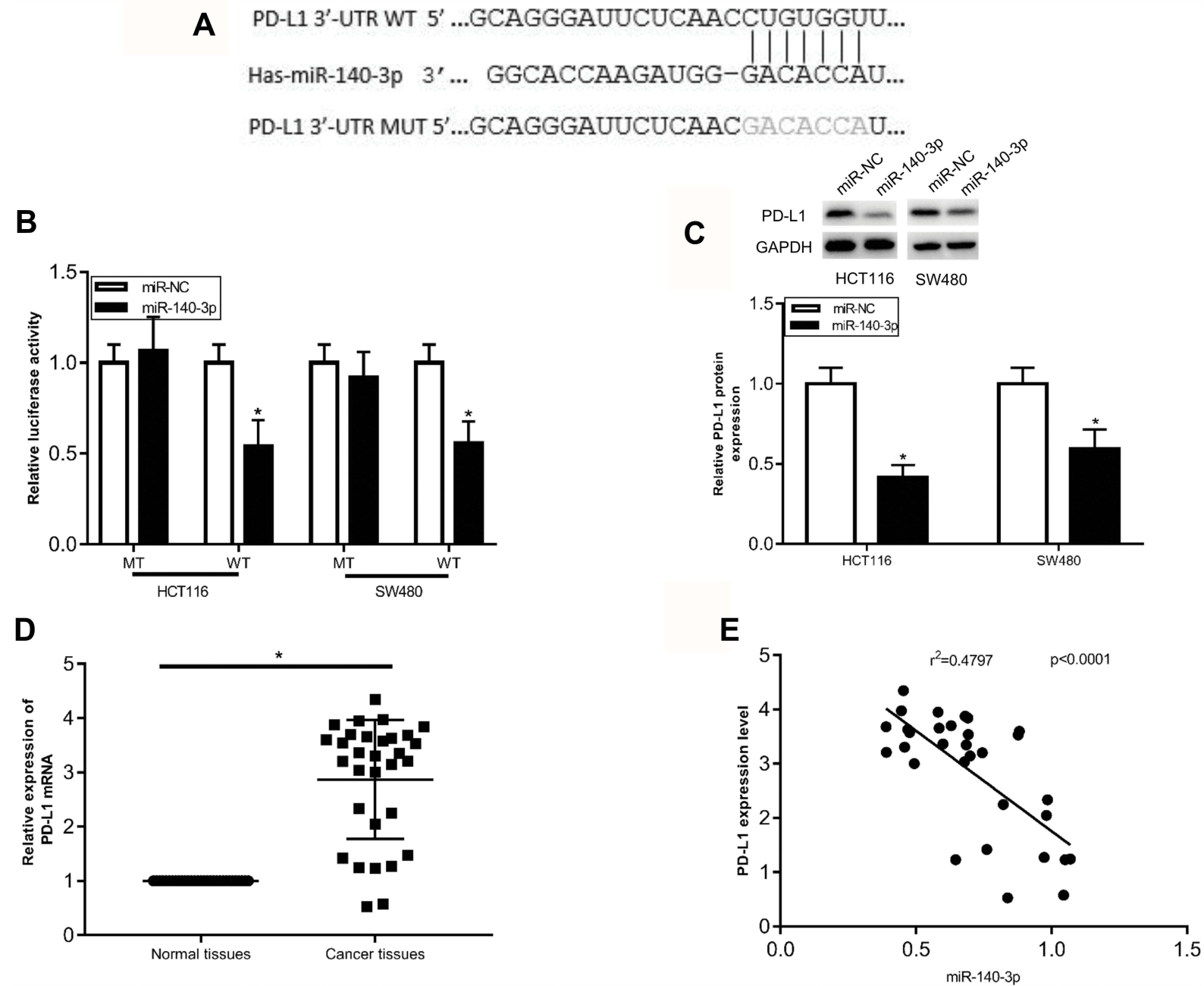

Figure 3 PD-LI is a direct target of miR-140-3p in CRC cells.

Notes: (A) Predicted binding sequences in the 3'UTR of PD-LI for miR-140-3p. The MUT binding sites in the 3'UTR of PD-LI for miR-I40-3p are also shown. (B) HCTI I6 and SW480 cells were co-transfected with a PD-LI 3'UTR (WT or MUT) luciferase reporter plasmid and miR-140-3p mimics or the miR-NC, then luciferase activity was determined. (C) PD-LI protein expression was determined in HCTII6 and SW480 cells transfected with miR-140-3p mimics or the miR-NC. (D) The mRNA expression of PD-LI was determined in 31 pairs of colorectal cancer and adjacent non-tumour tissues by RT-qPCR analysis. (E) The correlation between miR-140-3P and PD-LI expression in CRC tissues was determined using Spearman correlation analysis. $\left(r^{2}=0.4797, p<0.000 I, n=3 I\right) * P<0.05$.

Abbreviations: MUT, mutant type; WT, wild type;3'UTR, 3'-untranslated region; CRC, colorectal cancer; miR-NC, negative control; miRNA mimics PD-LI, programmed cell-death I ligand I.

\section{Overexpression Of PD-LI Attenuates The Tumour Suppressive Effect Of miR- 140-3p In CRC Cells}

The above experiments confirmed that miR-140-3p could play a vital role in inhibiting the progression of CRC by targeting PD-L1. A rescue experiment was used to corroborate these results. The PD-L1 overexpressing plasmid PCMV-PD-L1 or the empty plasmid PCMV was co-transfected with the miR-140-3p mimics into the HCT116 and SW480 cells. Western blot results showed that the expression level of PD-L1 was restored in the CRC cells transfected with PCMV-PD-L1 (Figure 5A). Figure 5B-D shows that the increase in PD-L1 expression enhanced the proliferation, migration and invasion ability of CRC cells. Moreover, the restoration of PD-L1 expression decreased the apoptosis of CRC cells (Figure 5E). These results further confirmed that miR-140-3p exerted its anticancer effect in CRC by inhibiting PD-L1.

\section{Upregulation Of miR-140-3p Inhibits Activation Of The PI3K/AKT Pathway In CRC}

Previous studies have reported that the loss of PTEN increases PD-L1 expression in many tumours by activating the PI3K/AKT pathway. ${ }^{17,18}$ Abnormal activation of the pathway is thought to be crucial for the occurrence and 


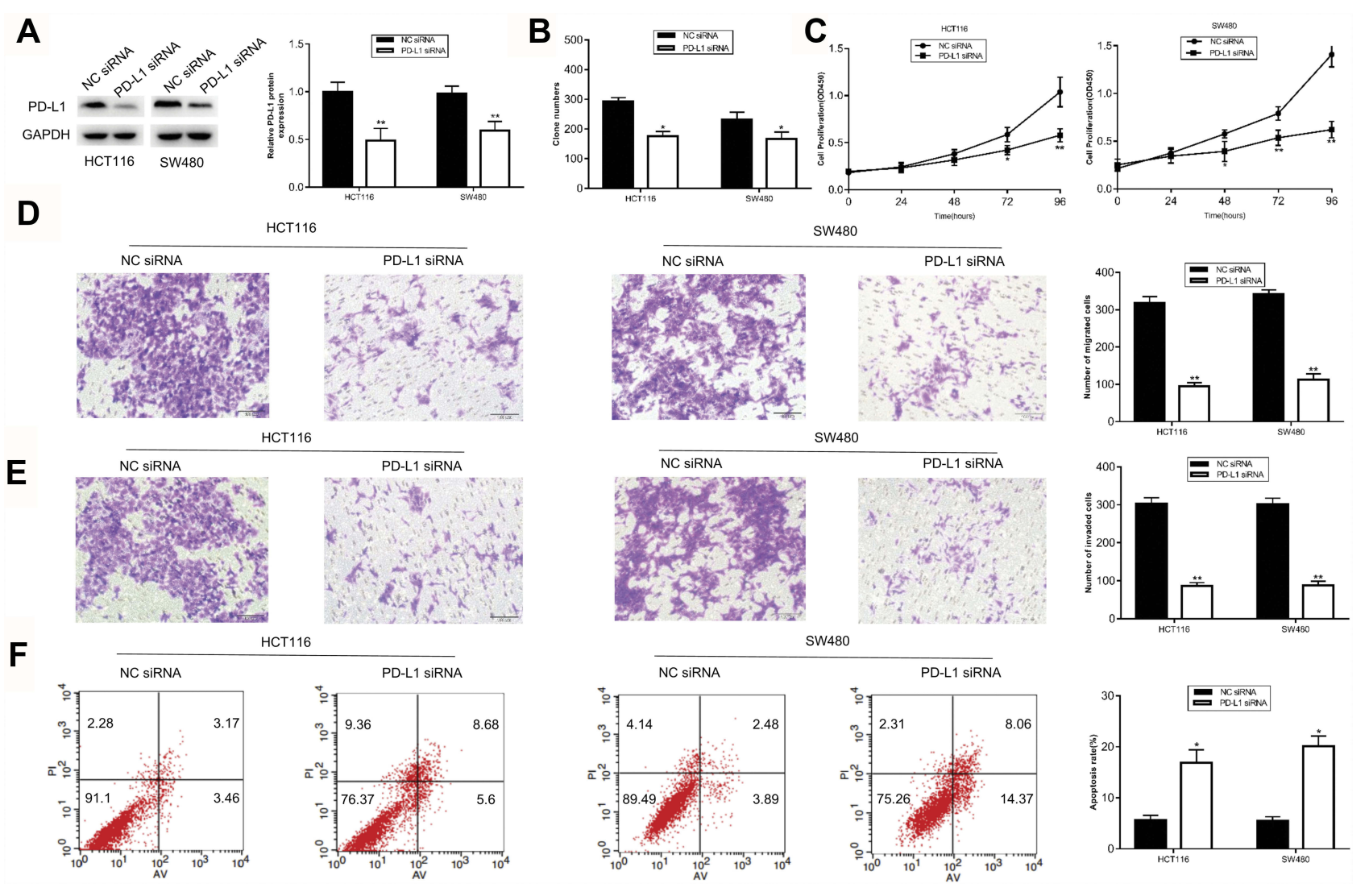

Figure 4 Knockdown of PD-LI suppresses the proliferation, inhibits the migration and invasion, and promotes the apoptosis of HCTII6 and SW480 cells.

Notes: (A) Western blot analysis was used to determine PD-LI protein expression in HCTII6 and SW480 cells transfected with PD-LI siRNA or NC siRNA. (B-E) The proliferation, migration and invasion capacities of HCTII6 and SW480 cells transfected with PD-LI siRNA or NC siRNA were detected by colony formation, CCK8, migration and invasion assays. (F) Flow cytometry was used to detect the effect of PD-LI inhibition on apoptosis of HCTII6 and SW480 cells transfected with PD-LI siRNA or NC siRNA. $* P<0.05, * * P<0.01$.

Abbreviations: CCK8, Cell counting kit8; miR-NC, negative control miRNA mimics; PD-LI, programmed cell-death I ligand I.

development of tumours. ${ }^{19,20}$ The above experimental data indicated that miR-140-3p directly targeted PD-L1 in CRC development. Thus, to further explore the potential mechanism by which miR-140-3p inhibited CRC development, we investigated $\mathrm{p}-\mathrm{AKT}$ and $\mathrm{p}-\mathrm{PI} 3 \mathrm{~K}$ expression after transfecting cells with miR-140-3p. As observed in Figure 6A, upregulation of miR-140-3p remarkably attenuated p-AKT and p-PI3K expression compared to the control group. In addition, HCT116 and SW480 cells transfected with PD-L1 siRNA had results that were similar to what was seen in the miR-140-3p treatment group (Figure 6A). Taken together, these data provide strong evidence that miR-140-3p may serve as a tumour suppressor in CRC development by disrupting the activation of the PI3K/AKT pathway.

\section{miR-140-3p Suppresses The Tumour Growth Of CRC In Vivo}

To determine the effects of miR-140-3p on tumour growth in vivo, subcutaneous xenograft models of CRC were constructed by implanting miR-140-3p or miR-NC transfected HCT116 cells into the right flank of nude mice. After 14 days, all nude mice were sacrificed and the tumour masses were weighed and measured. Nude mice injected with miR-140-3p transfected HCT116 cells exhibited a significantly smaller tumor weight and volume than what was seen in the mice transplanted with miR-NC transfected cells (Figure 7A-C). In addition, the expression of miR-140-3p in tumour tissues was detected by RT-qPCR. The results showed that miR-140$3 p$ expression was much higher in the miR-140-3p transfection group than it was in the control group (Figure 7D). Western blot analysis showed a significant decrease in PD-L1 expression in the miR-140-3p transfected group (Figure 7E). Furthermore, p-AKT and p-PI3K expression was also inhibited (Figure 7E). These data indicate that miR-140-3p suppressed tumour growth in vivo by targeting PD-L1 via inhibition of the PI3K/ AKT pathway. 


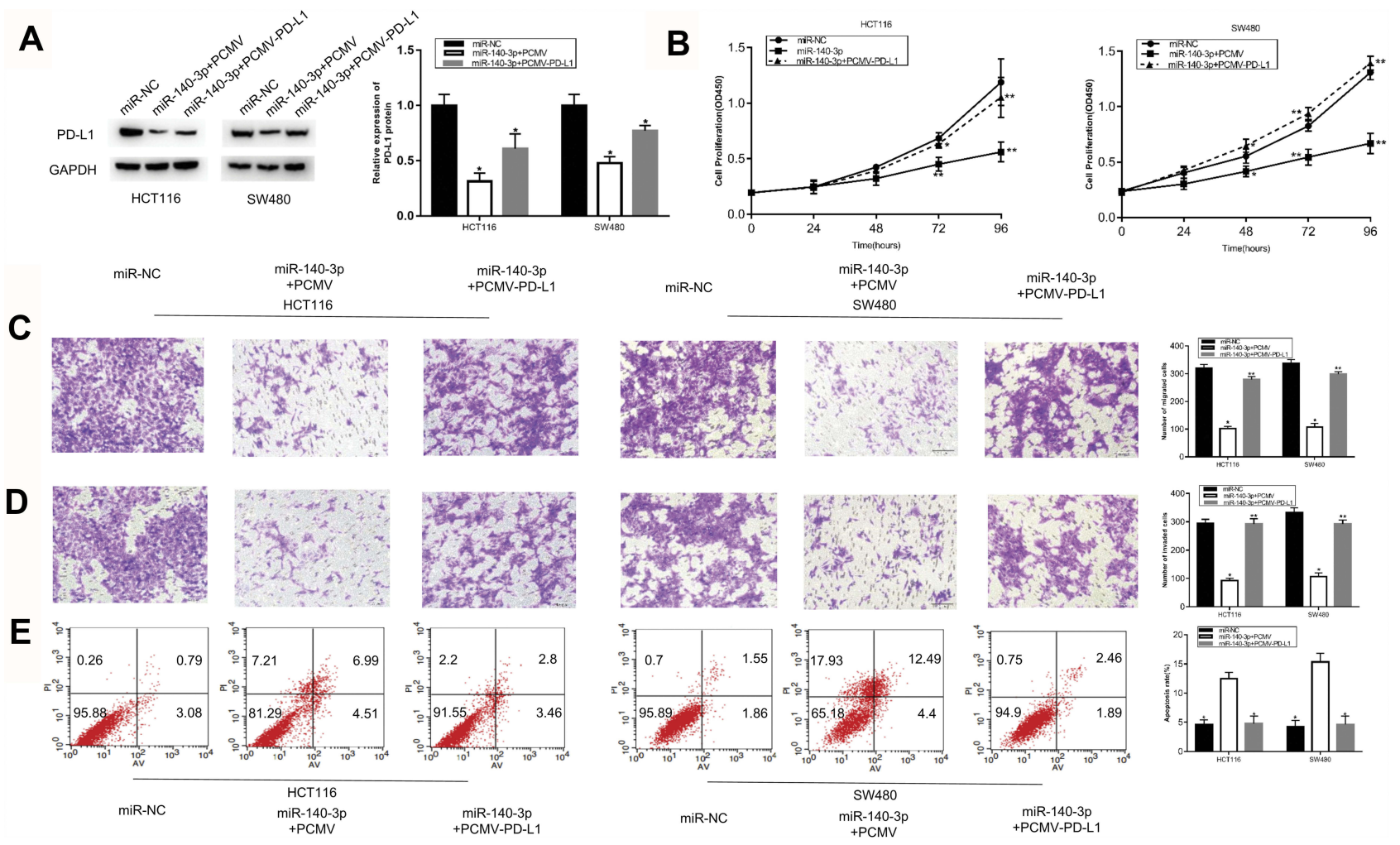

Figure 5 Restoration of PD-LI expression partially reverses the role of miR-I40-3P in CRC cells.

Notes: miR-140-3p mimics and the PCMV-PD-LI or empty PCMV plasmid were co-transfected into HCTII6 and SW480 cells, and the transfected cells were collected for subsequent experiments. (A) Western blot analysis was used to determine the protein level of PD-LI expression in different groups. (B-D) The proliferation, migration and invasion ability of HCTII6 and SW480 cells after transfection were detected by CCK8, migration and invasion assays. (E) Apoptosis of HCTII6 and SW480 cells after transfection was detected by flow cytometry. $* \mathrm{P}<0.05$, $* * \mathrm{P}<0.01$.

Abbreviations: PD-LI, programmed cell-death I ligand I; CCK8, Cell counting kit8.

A

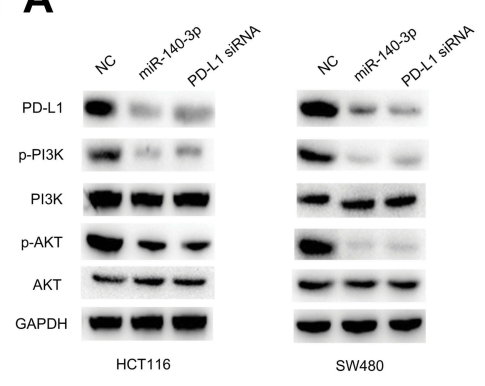

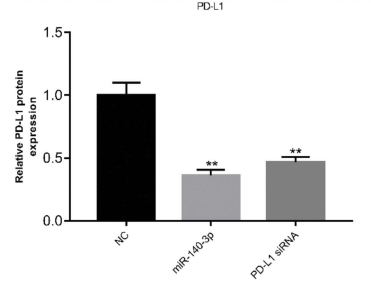

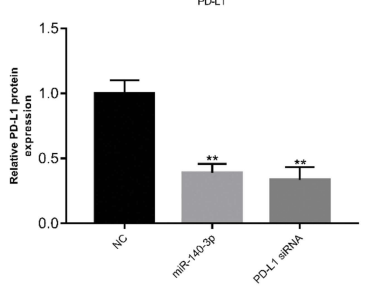

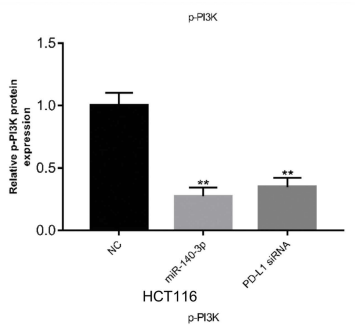

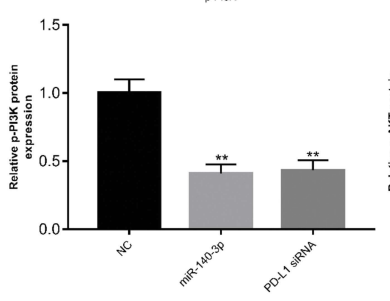

Sw480

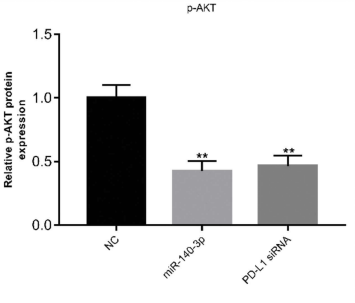

p-AKT

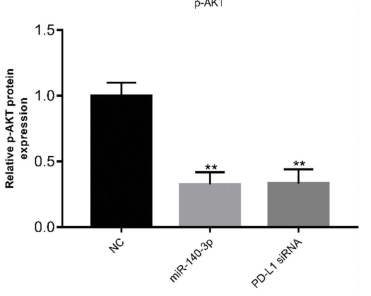

Figure 6 Suppression of miR-140-3p attenuates activation of the PI3K/AKT pathway.

Notes: (A) miR-I40-3p mimics, PD-LI siRNA or NC was transfected into HCTII6 and SW480 cells. The levels of proteins in the PI3K/AKT pathway were evaluated by using Western blot analysis. $* * * 0.01$.

Abbreviations: NC, negative control; PI3K: phosphatidylinositol 3-kinase; PD-LI, programmed cell-death I ligand I; AKT, protein kinase B; CCK8, Cell counting kit8.

\section{Discussion}

An increasing number of studies have indicated that miRNAs play an important regulatory role in the development and progression of CRC, making miRNAs critical for the diagnosis and prognosis of CRC. ${ }^{21,22}$ MiRNAs, such as comprising miR-139a-3p, miR-452, miR-449a and miR-205-5p have 
A

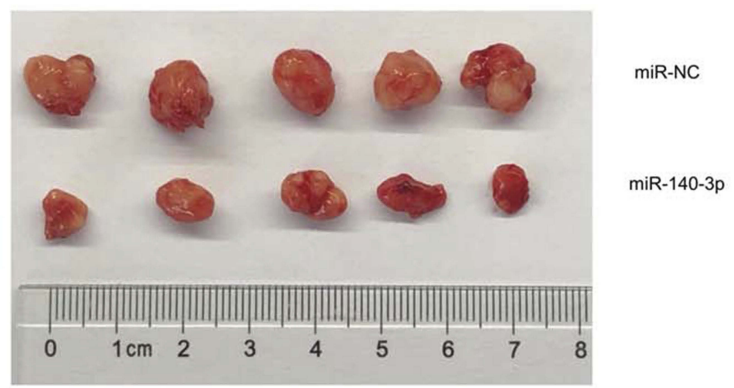

B

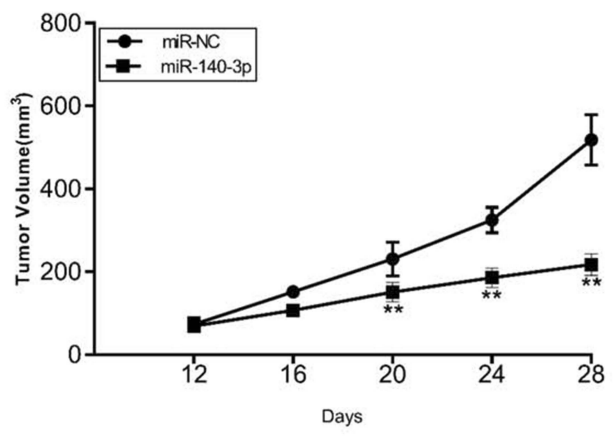

E

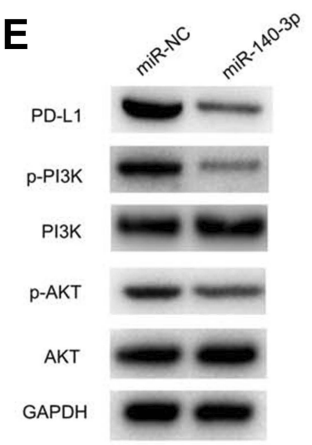

C

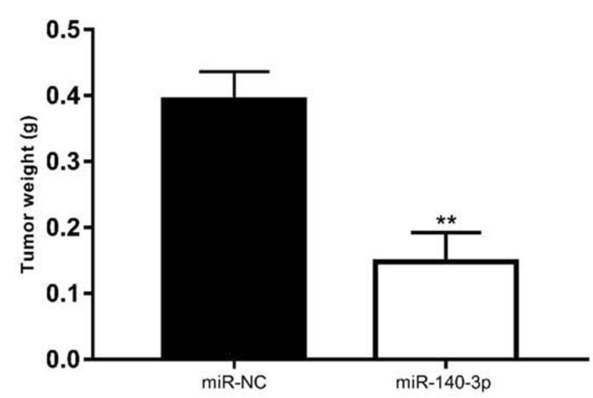

D

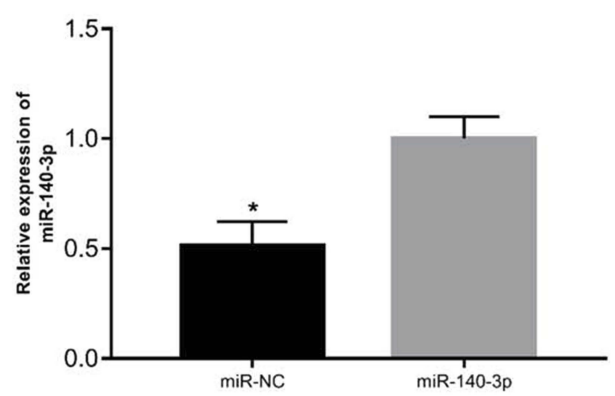

.


demonstrate that PD-L1 was a potential direct target of miR-140-3p. First, dual-luciferase reporter assays confirmed the bioinformatic analysis. Then, PD-L1 was overexpressed in CRC tissues, which was related to miR-140-3p expression. In addition, CRC cells transfected with PD-L1 siRNA exhibited a decrease in cell proliferative, migratory and invasive abilities. HCT116 and SW480 cells transfected with PD-L1 siRNA had the same biological behaviour as cells treated with miR-140-3p. Hence, these outcomes suggest that PD-L1 is a direct target of miR-140-3p and that miR-140-3p functions as a tumour suppressor by restricting PD-L1 expression.

The PI3K/AKT signalling pathway is confirmed to have a strong role in tumorigenesis. ${ }^{19,20}$ Previous studies have found positive feedback between AKT and PD-L1 expression. ${ }^{33}$ The dysfunction of the PI3K/AKT signalling pathway could regulate PD-L1 expression in lung cancer and breast cancer. ${ }^{35,36}$ In $\mathrm{CRC}$, it was found that downregulation of miR-140-3p is associated with activation of the AKT/mTOR signalling pathway. ${ }^{37}$ mTOR is also the downstream of PI3K/AKT signalling and plays a vital role in cellular growth and metabolism. ${ }^{38}$ In our research, p-AKT and p-PI3K expression was largely decreased in CRC cells treated with PD-L1 siRNA or miR-140-3p. More importantly, the outcome of Western blot analyses of tumour tissues from nude mice was nearly identical. These data suggest that the underlying mechanistic activity for miR-140-3p involves suppression in CRC progression.

In summary, our study demonstrated that miR-140-3p plays a pivotal role in the development and progression of CRC. Our experiments revealed that miR-140-3p regulated CRC cell proliferation, migration and apoptosis by directly targeting PD-L1. In addition, our study verified that the role of miR-140-3p in CRC could be mediated by PI3K/AKT inhibition. These findings suggest that miR$140-3 p$ is a potential new therapeutic target for CRC treatment.

\section{Acknowledgements}

This study was supported by the Primary Research \& Development Plan of Jiangsu Province grant (NO. BE2016799) and the Research Innovation Program for Graduates of Jiangsu Province grant (KYCX18-14).

\section{Author Contributions}

All authors made substantial contributions to data analysis, took part in drafting the article or revising it, gave final approval of the version to be published, and agree to be accountable for all aspects of the work.

\section{Disclosure}

The authors report no conflicts of interest in this work.

\section{References}

1. Bray F, Ferlay J, Soerjomataram I, et al. Global cancer statistics 2018: GLOBOCAN estimates of incidence and mortality worldwide for 36 cancers in 185 countries. CA Cancer J Clin. 2018;68:394-424. doi:10.3322/caac. 21492

2. Schreuders EH, Ruco A, Rabeneck L, et al. Colorectal cancer screening: a global overview of existing programmes. Gut. 2015;64:16371649. doi:10.1136/gutjnl-2014-309086

3. Hegde SR, Sun W, Lynch JP. Systemic and targeted therapy for advanced colon cancer. Expert Rev Gastroenterol Hepatol. 2008;2:135-149. doi:10.1586/17474124.2.1.135

4. Chen Y, Deng X, Chen W, et al. Silencing of microRNA-708 promotes cell growth and epithelial-to-mesenchymal transition by activating the SPHK2/AKT/beta-catenin pathway in glioma. Cell Death Dis. 2019;10:448. doi:10.1038/s41419-019-1671-5

5. Ji D, Chen Z, Li M, et al. MicroRNA-181a promotes tumor growth and liver metastasis in colorectal cancer by targeting the tumor suppressor WIF-1. Mol Cancer. 2014;13:86. doi:10.1186/14764598-13-86

6. Chen X, Zeng K, Xu M, et al. P53-induced miR-1249 inhibits tumor growth, metastasis, and angiogenesis by targeting VEGFA and HMGA2. Cell Death Dis. 2019;10:131. doi:10.1038/s41419-0181188-3

7. Ciesla M, Skrzypek K, Kozakowska M, et al. MicroRNAs as biomarkers of disease onset. Anal Bioanal Chem. 2011;401:2051-2061. doi:10.1007/s00216-011-5001-8

8. Dong W, Yao C, Teng X, et al. MiR-140-3p suppressed cell growth and invasion by downregulating the expression of ATP8A1 in nonsmall cell lung cancer. Tumour Biol. 2016;37:2973-2985. doi:10.1007/s13277-015-3452-9

9. Salem O, Erdem N, Jung J, et al. The highly expressed 5'isomiR of hsa-miR-140-3p contributes to the tumor-suppressive effects of miR140 by reducing breast cancer proliferation and migration. BMC Genomics. 2016;17:566. doi:10.1186/s12864-016-2869-x

10. Zou MX, Huang W, Wang XB, et al. Identification of miR-140-3p as a marker associated with poor prognosis in spinal chordoma. Int $J$ Clin Exp Pathol. 2014;7:4877-4885.

11. Li J, Zhao J, Wang H, et al. MicroRNA-140-3p enhances the sensitivity of hepatocellular carcinoma cells to sorafenib by targeting pregnenolone X receptor. Onco Targets Ther. 2018;11:5885-5894. doi:10.2147/OTT.S179509

12. Lee KS, Lee K, Yun S, et al. Prognostic relevance of programmed cell death ligand 1 expression in glioblastoma. $J$ Neurooncol. 2018;136:453-461. doi:10.1007/s11060-017-2675-6

13. Enwere EK, Kornaga EN, Dean M, et al. Expression of PD-L1 and presence of CD8-positive $\mathrm{T}$ cells in pre-treatment specimens of locally advanced cervical cancer. Mod Pathol. 2017;30:577-586. doi:10.1038/modpathol.2016.221

14. Liang M, Li J, Wang D, et al. T-cell infiltration and expressions of $\mathrm{T}$ lymphocyte co-inhibitory B7-H1 and B7-H4 molecules among colorectal cancer patients in northeast China's Heilongjiang province. Tumour Biol. 2014;35:55-60. doi:10.1007/s13277-013-1006-6

15. Droeser RA, Hirt C, Viehl CT, et al. Clinical impact of programmed cell death ligand 1 expression in colorectal cancer. Eur J Cancer. 2013;49:2233-2242. doi:10.1016/j.ejca.2013.02.015 
16. Frydenlund N, Mahalingam M. PD-L1 and immune escape: insights from melanoma and other lineage-unrelated malignancies. Hum Pathol. 2017;66:13-33. doi:10.1016/j.humpath.2017.06.012

17. Parsa AT, Waldron JS, Panner A, et al. Loss of tumor suppressor PTEN function increases B7-H1 expression and immunoresistance in glioma. Nat Med. 2007;13:84-88. doi:10.1038/nm1517

18. Stambolic V, Suzuki A, de la Pompa JL, et al. Negative regulation of PKB/Akt-dependent cell survival by the tumor suppressor PTEN. Cell. 1998;95(1):29-39. doi:10.1016/S0092-8674(00)81780-8

19. Dienstmann R, Rodon J, Serra V, et al. Picking the point of inhibition: a comparative review of $\mathrm{PI} 3 \mathrm{~K} / \mathrm{AKT} / \mathrm{mTOR}$ pathway inhibitors. Mol Cancer Ther. 2014;13:1021-1031. doi:10.1158/1535-7163.MCT-130639

20. Fruman DA, Rommel C. PI3K and cancer: lessons, challenges and opportunities. Nat Rev Drug Discov. 2014;13:140-156.

21. Moridikia A, Mirzaei H, Sahebkar A, et al. MicroRNAs: potential candidates for diagnosis and treatment of colorectal cancer. $J$ Cell Physiol. 2018;233:901-913. doi:10.1002/jcp.25801

22. Strubberg AM, Madison BB. MicroRNAs in the etiology of colorectal cancer: pathways and clinical implications. Dis Model Mech. 2017;10:197-214. doi:10.1242/dmm.027441

23. Takahashi H, Takahashi M, Ohnuma S, et al. microRNA-193a-3p is specifically down-regulated and acts as a tumor suppressor in BRAFmutated colorectal cancer. BMC Cancer. 2017;17:723. doi:10.1186/ s12885-017-3739-x

24. Li T, Jian X, He H, et al. MiR-452 promotes an aggressive colorectal cancer phenotype by regulating a Wnt/beta-catenin positive feedback loop. J Exp Clin Cancer Res. 2018;37:238. doi:10.1186/s13046-0180879-z

25. Ishikawa D, Takasu C, Kashihara H, et al. The significance of microRNA449a and its potential target HDAC1 in patients with colorectal cancer. Anticancer Res. 2019;39:2855-2860. doi:10.21873/anticanres.13414

26. Gulei D, Magdo L, Jurj A, et al. The silent healer: miR-205-5p upregulation inhibits epithelial to mesenchymal transition in colon cancer cells by indirectly up-regulating E-cadherin expression. Cell Death Dis. 2018;9:66. doi:10.1038/s41419-017-0102-8

27. Wienholds E, Kloosterman WP, Miska E, et al. MicroRNA expression in zebrafish embryonic development. Science. 2005;309:310 311. doi:10.1126/science. 1114519
28. Song B, Wang Y, Xi Y, et al. Mechanism of chemoresistance mediated by miR-140 in human osteosarcoma and colon cancer cells. Oncogene. 2009;28:4065-4074. doi:10.1038/onc.2009.274

29. Zhang W, Zou C, Pan L, et al. MicroRNA-140-5p inhibits the progression of colorectal cancer by targeting VEGFA. Cell Physiol Biochem. 2015;37:1123-1133. doi:10.1159/000430237

30. Ansell SM, Lesokhin AM, Borrello I, et al. PD-1 blockade with nivolumab in relapsed or refractory Hodgkin's lymphoma. $N$ Engl J Med. 2015;372:311-319. doi:10.1056/NEJMoa1411087

31. Bellmunt J, de Wit R, Vaughn DJ, et al. Pembrolizumab as secondline therapy for advanced urothelial carcinoma. $N$ Engl J Med. 2017;376:1015-1026. doi:10.1056/NEJMoa1613683

32. Overman MJ, Lonardi S, Wong KYM, et al. Durable clinical benefit with nivolumab plus ipilimumab in DNA mismatch repair-deficient/ microsatellite instability-high metastatic colorectal cancer. J Clin Oncol. 2018;36:773-779. doi:10.1200/JCO.2017.76.9901

33. Wang X, Li J, Dong K, et al. Tumor suppressor miR-34a targets PD$\mathrm{L} 1$ and functions as a potential immunotherapeutic target in acute myeloid leukemia. Cell Signal. 2015;27:443-452. doi:10.1016/j. cellsig.2014.12.003

34. Yang L, Cai Y, Zhang D, et al. miR-195/miR-497 regulate CD274 expression of immune regulatory ligands in triple-negative breast cancer. J Breast Cancer. 2018;21:371-381. doi:10.4048/jbc. 2018.21.e60

35. Zhang X, Zeng Y, Qu Q, et al. PD-L1 induced by IFN-gamma from tumor-associated macrophages via the JAK/STAT3 and PI3K/AKT signaling pathways promoted progression of lung cancer. Int J Clin Oncol. 2017;22:1026-1033. doi:10.1007/s10147-017-1161-7

36. Almozyan S, Colak D, Mansour F, et al. PD-L1 promotes OCT4 and Nanog expression in breast cancer stem cells by sustaining PI3K/ AKT pathway activation. Int $J$ Cancer. 2017;141:1402-1412. doi:10.1002/ijc.v141.7

37. Wu SM, Li TH, Yun H, et al. miR-140-3p knockdown suppresses cell proliferation and fibrogenesis in hepatic stellate cells via PTENmediated AKT/mTOR signaling. Yonsei Med J. 2019;60:561-569. doi:10.3349/ymj.2019.60.6.561

38. Laplante M. Sabatini DM: mTOR signaling in growth control and disease. Cell. 2012;149:274-293. doi:10.1016/j.cell.2012.03.017
OncoTargets and Therapy

\section{Publish your work in this journal}

OncoTargets and Therapy is an international, peer-reviewed, open access journal focusing on the pathological basis of all cancers, potential targets for therapy and treatment protocols employed to improve the management of cancer patients. The journal also focuses on the impact of management programs and new therapeutic

Submit your manuscript here: https://www.dovepress.com/oncotargets-and-therapy-journa agents and protocols on patient perspectives such as quality of life, adherence and satisfaction. The manuscript management system is completely online and includes a very quick and fair peer-review system, which is all easy to use. Visit http://www.dovepress.com/ testimonials.php to read real quotes from published authors. 